TITLE:

\title{
Toward photochemistry of integrated heterogeneous systems.
}

$\operatorname{AUTHOR}(S)$ :

Matsumoto, Yoshiyasu

CITATION:

Matsumoto, Yoshiyasu. Toward photochemistry of integrated

heterogeneous systems.. The Journal of chemical physics 2012, 137(9):

091705.

ISSUE DATE:

2012-09

URL:

http://hdl.handle.net/2433/160366

RIGHT:

(C) 2012 American Institute of Physics 


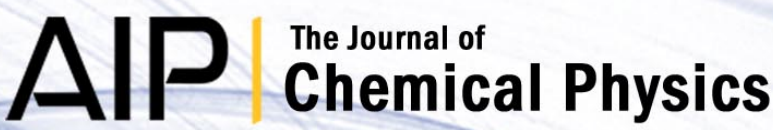

\section{Toward photochemistry of integrated heterogeneous systems}

Yoshiyasu Matsumoto

Citation: J. Chem. Phys. 137, 091705 (2012); doi: 10.1063/1.4746802

View online: http://dx.doi.org/10.1063/1.4746802

View Table of Contents: http://jcp.aip.org/resource/1/JCPSA6/v137/i9

Published by the American Institute of Physics.

\section{Additional information on J. Chem. Phys.}

Journal Homepage: http://jcp.aip.org/

Journal Information: http://jcp.aip.org/about/about_the_journal

Top downloads: http://jcp.aip.org/features/most_downloaded

Information for Authors: http://jcp.aip.org/authors

\section{ADVERTISEMENT}

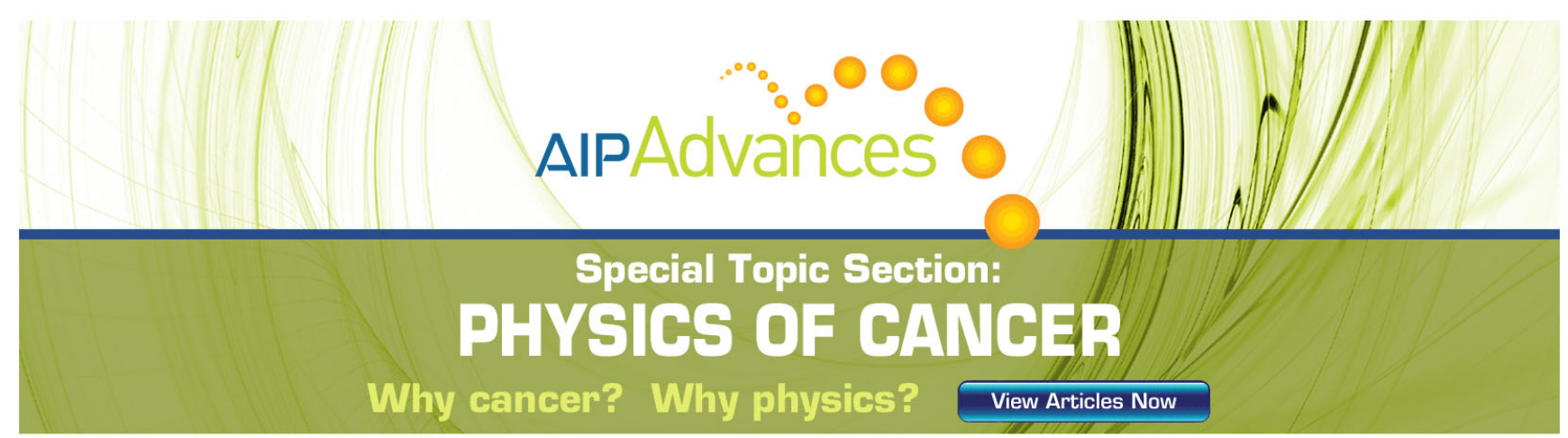




\title{
Toward photochemistry of integrated heterogeneous systems
}

\author{
Yoshiyasu Matsumoto ${ }^{\text {a) }}$ \\ Kyoto University, Graduate School of Science, Department of Chemistry, Kyoto 606-8502, Japan
}

(Received 3 February 2012; accepted 11 May 2012; published online 4 September 2012)

\begin{abstract}
This paper begins with describing the excitation mechanisms in surface photochemistry and nuclear dynamics of adsorbate induced by electronic excitation. An illustrative example is Cs adsorbate on a $\mathrm{Cu}(111)$ surface. This adsorption system shows drastic changes in the electronic structure with coverage; this allows us to examine different types of electronic excitations that stimulate nuclear motions of Cs. Remarks are made on challenges in photoinduced processes at well-defined surfaces: direct observations of adsorbate-substrate vibrational modes and photoinduced reactions between adsorbates. Then, the paper addresses some issues in more complex systems: metal-liquid interfaces and powdered photocatalysts of metal oxides. Photochemistry and photoinduced nuclear dynamics at metal-liquid interfaces have not been well explored. Studies on this subject may make it possible to bridge the gap between surface photochemistry and electrochemistry. Photocatalysis with powdered catalysts has been extensively studied and is still an active area, but our understanding of the mechanism of photocatalysis is far from satisfactory. Although complicated, the highly integrated systems provide an opportunity to extend our knowledge of surface photochemistry. (C) 2012 American Institute of Physics. [http://dx.doi.org/10.1063/1.4746802]
\end{abstract}

\section{INTRODUCTION}

Because metal has continuum electronic bands, nuclear motions of adsorbate are associated with electronic excitation and de-excitation in metal. This type of nonadiabatic coupling was not familiar to a spectroscopist who had been working on electronic and vibrational spectroscopy of molecules and complexes isolated in the gas phase. In addition, the knowledge and experience gained in the field of radiationless transitions of electronically excited molecules made me think that the lifetime of the excited state should be extremely short if molecules are close proximity to metal surfaces. Thus, I was surprised by the fact that photochemistry of adsorbates including photo-stimulated desorption takes place upon valence electron excitation at metal surfaces. Since then, I have been fascinated by the photochemistry and photoinduced processes at metal surfaces for more than two decades.

As in the early works on photochemistry in homogeneous media: gas and liquid phases, a primary objective in the studies of surface photochemistry was product analysis by using conventional surface science techniques: temperatureprogrammed desorption, x-ray photoelectron spectroscopy, infrared reflection absorption spectroscopy, high-resolution electron energy loss spectroscopy, etc. More detailed information of desorbed species, such as energy distributions among translation, rotation, vibration, and electron spin, was also obtained by applying the sensitive detection techniques developed in gas phase spectroscopy: laser-induced fluorescence and resonant enhanced multiphoton ionization. Measurements of angular distributions of desorbed species were also useful to probe the potential energy surface (PES) for molecules departing from the surface. Introduction of ultrafast lasers opened a new field of surface science, i.e., ultrafast sur-

\footnotetext{
a)matsumoto@kuchem.kyoto-u.ac.jp.
}

face dynamics. Substantial developments made by mid 1990s were collected in the monograph edited by Dai and Ho. ${ }^{1}$

One of the biggest questions in surface photochemistry at metal surfaces has been excitation mechanisms: what type of electronic excitation is responsible for the photochemistry? We cannot irradiate exclusively adsorbate nor substrate with photons. Thus, we always have to ask ourselves what we are really exciting. Figure 1 shows two idealized excitation mechanisms frequently discussed in the surface photochemistry community: (a) substrate-mediated indirect excitation and (b) adsorbate-localized direct excitation. For example, we were puzzled why methane weakly adsorbed on $\mathrm{Pt}(111)$ is dissociated with $6 \mathrm{eV}$ photons where no absorption bands of methane exist in the gas phase. ${ }^{2}$ Since platinum absorbs $6-\mathrm{eV}$ photons, we tended to postulate that the photochemistry occurs via substrate-mediated excitation. But, the incidence angle dependence of photochemical yields upon the irradiation of a linearly polarized light contradicts this mechanism; it indicates that the excitation takes place with a transition dipole orienting along the surface normal. ${ }^{3,4}$ We proposed the direct excitation mechanism from the highest occupied molecular orbital (HOMO) state to an excited state of methane hybridized with substrate electronic states. ${ }^{4-6}$ Later, this mechanism was proved to be right with $\mathrm{x}$-ray absorption spectroscopy performed by Nilsson and co-workers; ${ }^{7}$ they found new adsorption-induced bands right above the Fermi level that can be accessible from HOMO of methane with a $6-\mathrm{eV}$ photon. Since we studied the photochemistry of methane, the question on the excitation mechanism has been my central concern in surface photochemistry at metal surfaces.

This paper certainly does not aim to review extensively the photochemistry at metal surfaces. In Sec. II, I will discuss the excitation mechanisms and photoinduced nuclear dynamics by using an illustrative example, Cs adsorbed on $\mathrm{Cu}(111)$. Then, I will describe my personal perspective on 


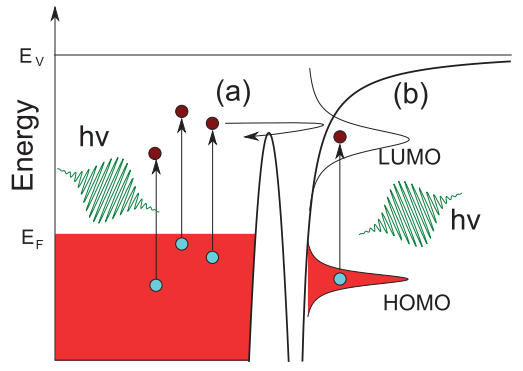

FIG. 1. Excitation mechanisms in photochemistry at metal surfaces: (a) Substrate-mediated indirect excitation and (b) adsorbate-localized direct excitation.

photoinduced surface dynamics at well-defined adsorption systems in Sec. III and beyond, i.e., photochemistry and photocatalysis of more complex, and highly integrated heterogeneous systems in Sec. IV.

\section{EXCITATION MECHANISM AND NUCLEAR DYNAMICS: AN ILLUSTRATIVE EXAMPLE}

The electronic structure of alkali-covered metal surfaces has been one of the central issues in surface science and has been extensively studied. ${ }^{8}$ Figure 2 illustrates schematically a typical electronic structure of alkali adsorption systems as a function of coverage, based on the experimental and theoretical works on $\mathrm{Na}$ (Refs. 9 and 10) and K (Ref. 11) on metal surfaces. One of the advantages using a $\mathrm{Cu}(111)$ surface as a substrate is that a two-dimensional potential well is formed between the substrate and vacuum barriers at this surface as a result of the $s, p$-inverted $L$-projected bandgap characteristic of a clean $\mathrm{Cu}(111)$ surface. What is fascinating in the alkali adsorption system is that we can tune the electronic structure by changing alkali coverage.

At the low coverage limit, an alkali-induced electronic state, absent on the clean surface, appears at an energy be-

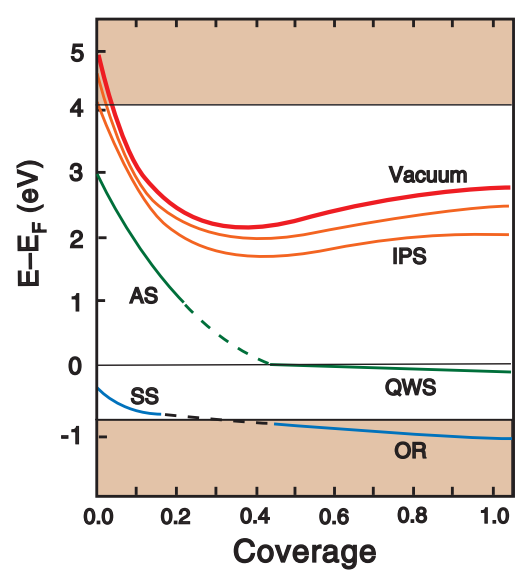

FIG. 2. Electronic states on an alkali-covered $\mathrm{Cu}(111)$ surface as a function of coverage. IPS: image potential state, AS: antibonding state, SS: Shockley surface state, QWS: quantum-well state, and OR: overlayer resonance. The shaded regions show the band structure of bulk copper projected onto the surface Brillouin zone. The energy structures are adapted from the experimental and theoretical works on $\mathrm{Na}$ (Refs. 9 and 10) and $\mathrm{K} .{ }^{11}$ Because this figure is to provide the electronic structure qualitatively, the energies plotted here are not exact. tween the image potential states (IPSs) and the Fermi level $E_{\mathrm{F}}$. This state correlates to the electronic levels of an isolated alkali atom in vacuum: the occupied outermost $s$ and unoccupied $p_{z}$ levels. As the alkali atom approaches to the metal surface, the $s$ and $p_{z}$ orbitals are hybridized as a result of interaction with the metal to form a nonstationary state. ${ }^{12}$ Because the wave function of this state has an antibonding character with respect to the alkali-substrate bond, this state is denoted as the antibonding state (AS). This antibonding state is located at $\sim 3 \mathrm{eV}$ above $E_{\mathrm{F}}$ irrespective of the alkali-atom period, ${ }^{13}$ steeply shifting toward $E_{\mathrm{F}}$ with increasing coverage. The Shockley surface state (SS) also shifts downward with increasing coverage and it crosses the lower energy edge of the $L$-band gap.

At high coverages, the alkali overlayers are metallic, because the orbitals of neighboring alkali atoms overlap extensively with each other. Two bands characteristic of the overlayer are an overlayer resonance (OR) located below the $L$-band gap and a quantum well state (QWS) at around $E_{\mathrm{F}}$. These bands correlate to those of a free-standing alkali monolayer in the vacuum: the $s$-like lowest and the $p_{z}$-like second lowest bands. ${ }^{14}$ When the monolayer is brought closer to the metal surface, they are stabilized by the interaction with the metal, while these bands maintain the integrity. The $s$-like band correlates to $\mathrm{OR}$; the $p_{z}$-like band correlates to QWS. Because QWS is located in the $L$-band gap, its wave function is localized at the surface. In contrast, the wave function of OR extends more into the substrate, because it is located below the lower edge of $L$-band gap.

In both coverage ranges, the dynamics of $\mathrm{Cs}$ adsorbate associated with specific electronic excitations have been explored. At the low coverages, Petek and co-workers probed the nuclear motions of Cs adsorbates in an electronic excited state using interferometric time-resolved two-photon photoemission (2PPE). ${ }^{15,16}$ 2PPE spectra as a function of Cs coverage clearly show a resonance feature. Thus, the excitation pathway is clearly defined: the resonant transition from SS to AS. A Cs adatom rests on the ionic PES of the ground electronic state before photo-irradiation. When an ultrafast pump pulse resonantly excites the adsorbate system via the transition from SS to AS, Cs is excited to a repulsive PES; thus, it starts to move along the surface normal. However, the lifetime of the excited state is so short that the Cs atom is quenched back to the deep ionic PES; thus, it cannot desorb. This photoinduced process is called "frustrated" desorption.

At the high coverages, our group explored the nuclear dynamics, i.e., coherent stretching motions of $\mathrm{Cs}$ induced by electronic transition, using time-resolved second harmonic generation (TRSHG). ${ }^{17,18}$ Figure 3(a) shows typical traces of TRSHG signals. Both excitations at the wavelengths $\lambda_{\mathrm{ex}}$ $=400$ and $800 \mathrm{~nm}$ generate coherent surface phonons of Cs-Cu stretching at $v=1.8 \mathrm{THz}$.

Although there is little difference in frequency, the initial phase of the oscillating component at $\lambda_{\mathrm{ex}}=800 \mathrm{~nm}$ is very different from that at $\lambda_{\mathrm{ex}}=400 \mathrm{~nm}$, as shown in Fig. 3(b). The initial phase provides us the information of the temporal profile of force driving the coherent vibration. A classical forced harmonic oscillator of a frequency $\Omega_{0}$ with a damping 

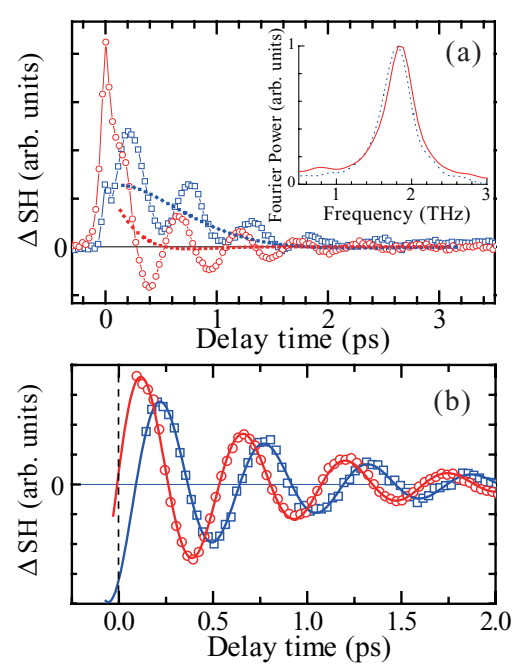

FIG. 3. (a) Excitation wavelength dependence of TRSHG traces taken from $\mathrm{Cs} / \mathrm{Cu}(111)$ at $\lambda_{\mathrm{ex}}=800 \mathrm{~nm}$ (red open circles) and $\lambda_{\mathrm{ex}}=400 \mathrm{~nm}$ (blue open squares). The probe wavelength is $565 \mathrm{~nm}$ for both traces. The incident pump fluence was $8.0 \mathrm{~mJ} / \mathrm{cm}^{2}$ and the Cs coverage was $0.23 \mathrm{ML}$ at $\lambda_{\text {ex }}$ $=800 \mathrm{~nm} ; 3.6 \mathrm{~mJ} / \mathrm{cm}^{2}$; and $0.25 \mathrm{ML}$ at $\lambda_{\mathrm{ex}}=400 \mathrm{~nm}$. Solid lines are results of the nonlinear least-square fitting with Eq. (1). Dotted lines are overdamped components contributed by hot electrons. (Inset) Fourier power spectra of the oscillatory components of the TRSHG traces for $\lambda_{\mathrm{ex}}=800 \mathrm{~nm}$ (solid) and for $\lambda_{\mathrm{ex}}=400 \mathrm{~nm}$ (dotted). (b) Oscillating components obtained by subtracting the overdamped ones. Note that initial phases are very different to each other, while the frequencies are very similar. Reprinted with permission from K. Watanabe, Y. Matsumoto, T. Yasuike, and K. Nobusada, J. Phys. Chem. A 115, 9528 (2011). Copyright 2011 American Chemical Society.

rate $\beta$ results in a decaying oscillation

$$
Q(t) \propto \cos \left(\Omega_{1} t-\phi\right) e^{-\beta t},
$$

where $\Omega_{1}=\sqrt{\Omega_{0}^{2}-\beta^{2}}$ and $\phi$ is the initial phase. If the force is impulsive, i.e., $F(t) \propto \delta(t)$, the initial phase is $\pi / 2$ : the nuclear oscillation is described as $Q(t) \propto \exp (-\beta t) \sin \Omega_{1} t$. If the force has a temporal profile of a step function, $F(t) \propto \theta(t)$, the initial phase is 0 or $\pi$. Fitting of the TRSHG traces to Eq. (1) determined the initial phases: $\phi=(-84 \pm 1)^{\circ}$ at $\lambda_{\mathrm{ex}}$ $=800 \mathrm{~nm}$ and $(-149 \pm 1)^{\circ}$ at $\lambda_{\mathrm{ex}}=400 \mathrm{~nm}$. Thus, $\mathrm{Cs}-\mathrm{Cu}$ stretching is sin-like at $\lambda_{\mathrm{ex}}=800 \mathrm{~nm}$, while it is close to coslike at $\lambda_{\text {ex }}=400 \mathrm{~nm}$.

Let us discuss what electronic transitions are responsible for this distinct excitation wavelength dependence of initial phase. The electron density around a $\mathrm{Cs}$ adatom has to be modulated to generate the force field driving its coherent stretching motion. The electronic transitions involved in adsorbate-induced electronic states are effective in doing so. The unoccupied Cs $5 d$ band was reported to be at $\sim 1.6 \mathrm{eV}$ in inverse photoemission measurements. ${ }^{19}$ Furthermore, according to density functional theory calculations, ${ }^{20}$ the third IPS at $\bar{\Gamma}$ is also located at $\sim 1.6 \mathrm{eV}$. Because QWS is located near $E_{\mathrm{F}}$, the transitions from QWS to these unoccupied surface localized states are near resonant to the photon energy at $\lambda_{\mathrm{ex}}=800 \mathrm{~nm}: \mathrm{h} v=1.55 \mathrm{eV}$. Although 800-nm photons are absorbed by the substrate, the oscillator strength of transition between adsorbate-localized states from QWS to the unoccupied surface band originating in the $5 d$ band of Cs or to the third IPS could be substantially larger than the bulk transitions in the $s, p$-band, because the former transitions are near resonant at $800 \mathrm{~nm}$. In this case, Cs is excited locally and quenched very rapidly. Thus, the excited lifetime is very short. Consequently, the force exerted to Cs is regarded as impulsive compared with a period of $\mathrm{Cs}-\mathrm{Cu}$ stretching, resulting in the sin-like oscillation.

In contrast, substrate excitation is more substantial at $\lambda_{\mathrm{ex}}$ $=400 \mathrm{~nm}$ than at $\lambda_{\text {ex }}=800 \mathrm{~nm}$, because $400-\mathrm{nm}$ photons can excite electrons in the $d$-bands of $\mathrm{Cu}$ in addition to those in the $s, p$-band. Upon excitation by fs pump pulses with a fluence of $20 \mathrm{~mJ} / \mathrm{cm}^{2}$ at $\lambda_{\mathrm{ex}}=400 \mathrm{~nm}$, the substrate electron temperature increases to several thousand $\mathrm{K}$ within $0.1 \mathrm{ps}$ and decays to $1000 \mathrm{~K}$ in $\sim 1$ ps. Electron transfer from the substrate to the adsorbate-localized states takes place, while a bath of substrate electrons maintains its temperature high enough for electrons to transfer to the adsorbate unoccupied states. This implies that the electron temperature increases with a very sharp rising edge and stays high in a much longer time interval than a period of $\mathrm{Cs}-\mathrm{Cu}$ stretching. Thus, the force exerted to $\mathrm{Cs}$ has a temporal profile close to a step function. Consequently, the substrate excitation is believed to be responsible for generation of coherent phonons with the cos-like oscillation.

This illustrative example, $\mathrm{Cs}$ atoms on $\mathrm{Cu}(111)$, shows that the alkali-induced electronic states play an central role in generating photoinduced coherent nuclear motions of adsorbates: AS for the "frustrated" desorption in low coverages on the one hand, and QWS for the coherent phonons of the Cs stretching mode in high coverages on the other. This adsorption system is one of the few examples where the detailed discussion on how adsorbate nuclear motions are induced with a specific electronic excitation, since the electronic structure is relatively well known over a wide range of coverage.

\section{CHALLENGES IN PHOTOCHEMISTRY OF WELL-DEFINED ADSORPTION SYSTEMS}

\section{A. Ultrafast dynamics of adsorbate-substrate vibrational modes}

Excitation of adsorbate-substrate vibrational modes (referred to external modes hereafter) influences many physical and chemical processes at surfaces, including charge transfer between adsorbate and substrate and surface chemistry particularly for Langmuir-Hinshelwood type reactions. In spite of the important roles of external modes, the dynamics of external modes have not been explored extensively. The illustrative example described in Sec. II shows that ultrafast electronic excitation at surfaces can generate coherent motions of adsorbate with respect to the surface. This possibility is not limited to adatoms; the external modes of polyatomic adsorbates can also be excited by impulsive electronic excitation.

Nonadiabatic coupling between substrate electrons and nuclear motions is deeply involved in both the excitation and relaxation of adsorbate vibrational modes including external modes. The important role of nonadiabatic coupling at metal surfaces has been postulated ${ }^{21,22}$ and thoroughly studied in the studies of vibrational relaxation of internal stretching modes, ${ }^{23-27}$ in particular, carbon monoxide: a molecular 
adsorbate most extensively studied in surface science. Adsorbates are desorbed effectively under intense excitation of substrate electrons by fs laser pulses. This is also a manifestation of nonadiabatic coupling; substrate electrons transfer energy to adsorbate nuclear motions particularly to external modes, resulting in desorption of adsorbates. Desorption yields depend on the fluence of pump pulses nonlinearly. Depending on the energy of an unoccupied state, the fs-laser induced desorption can be accounted for by the model of electronic friction or desorption induced by multiple electronic excitation. ${ }^{28}$ Diffusion of adsorbates is also stimulated by the intense substrate excitation by fs laser pulses. Bonn and co-workers have wisely used a stepped surface of platinum to show that diffusion of $\mathrm{CO}$ is effectively promoted by excitation of the frustrated rotational mode. ${ }^{29}$ Thus, the nonadiabatic coupling plays a key role in photoinduced nuclear dynamics at metal surfaces. One of the manifestations of nonadiabatic coupling in spectral features is Fano-like line shapes predicted by Langreth. ${ }^{22}$ By using infrared-visible sum frequency generation with heterodyne detection, we have recently demonstrated that the $\mathrm{C}-\mathrm{O}$ stretching band of $\mathrm{CO}$ on $\mathrm{Pt}(111)$ shows a Fano-like line shape in an imaginary part of the second-order nonlinear susceptibility right after fs laser excitation. ${ }^{30}$

These observations and theoretical analyses have advanced our understanding of nonadiabatic coupling between substrate electrons and adsorbate motions. However, the dynamics of external modes excited by electronic transitions have been monitored indirectly through the changes in spectral features of the internal mode, such as $\mathrm{C}-\mathrm{O}$ stretching, due to anharmonic coupling with frustrated modes. Direct observations of the dynamics of external modes initiated by electronic excitation are still lacking. These observations will further deepen our understanding of nonadiabatic coupling and adsorbate nuclear dynamics, and may lead to the first step for manipulation of surface chemistry. It is also inevitable that extensive theoretical calculations provide multi-dimensional potential energy surfaces with high precision.

\section{B. Surface photochemistry}

Most studies on surface photochemistry have been devoted to desorption and dissociation of adsorbate. There have been a few works focusing on reactions between adsorbates: $\mathrm{CO}$ oxidation on $\mathrm{Pt}$ coadsorbed with $\mathrm{O}_{2},{ }^{31,32} \mathrm{CO}$ oxidation on $\mathrm{Ru}(0001)$ with oxygen atoms, ${ }^{33}$ oxygen recombination on $\mathrm{Pt}(111)$ with $\mathrm{O}$ and $\mathrm{N}_{2} \mathrm{O}$ coadsorbates, ${ }^{34}$ and associative desorption of hydrogen. ${ }^{35}$ Recently, Gerber and co-workers have demonstrated that $\mathrm{CO}$ and hydrogen coadsorbed on $\mathrm{Pd}(100)$ produce various molecules including formaldehyde, water, etc., upon the irradiation of fs laser pulses. ${ }^{36,37}$ In addition, they showed that the product yield ratio can be optimized by changing the time profile of excitation pulses. Definitely, we need more examples of photoinduced chemistry among adsorbates. Moreover, it is important to detect reaction intermediates in the surface photochemistry for a better understanding of the reaction mechanisms.

One of the obstacles to detecting intermediates by timeresolved measurements is that surface conditions may change during acquisition times; for example, photochemical products may be accumulated at a surface, which may poison reaction sites. This is very different from time-resolved measurements performed in the gas and liquid phases. In the homogeneous systems, samples can be easily refreshed by using a flow reactor such that reactants are supplied and products are eliminated continuously. At surfaces, it is not easy to do so. One way to avoid the accumulation of products is to move a sample surface such that laser pulses are always directed to fresh surface area during measurements. Other possibility is to focus on photocatalytic processes, where a stationary state can be maintained during measurements by balancing the rates of adsorption of reactants and desorption of products.

\section{TOWARD INTEGRATED HETEROGENEOUS SYSTEMS}

\section{A. Photochemistry at solid-liquid interfaces}

Although much progress has been made in the studies on photoinduced nuclear dynamics at surfaces in ultrahigh vacuum (UHV), the dynamics at metal-liquid interfaces are still little known and waiting to be explored.

First, I like to point out some similarity in concept between substrate-mediated excitation described earlier and electrochemistry. As shown in Fig. 1, photo-irradiation generates nonthermal substrate electron distributions transiently. In the substrate-mediated excitation at metal surfaces covered with adsorbate in vacuum, charge transfer between adsorbate and substrate triggers surface reactions: substrate electrons (holes) energetically high enough can be transferred to an adsorbate unoccupied (occupied) state. Such charge-transfer driven surface reactions are ubiquitous in electrochemistry.

In electrochemistry, an external bias voltage is applied to a metal electrode; this shifts its Fermi level with respect to the energy levels of adsorbate. For example, when the metal electrode is negatively biased such that the Fermi level is aligned to an adsorbate unoccupied state, electrons at the Fermi level in the metal electrode can be transferred to the unoccupied state: the reduction of adsorbate takes place. In this way, electron or hole transfer rates are controlled by external bias voltage. Surface photochemistry of adsorbate is induced by irradiating a metal surface with photons creating hot electrons, whereas reactions are induced in electrochemistry by applying external bias to an electrode to change the energy of substrate electrons relative to adsorbate electronic states. Thus, photochemistry induced by substrate-mediated excitation has some analogy with electrochemistry.

Following this analogy, one might think that ultrafast nuclear dynamics discussed earlier can be explored by applying an ultrafast electric pulse to an electrode. However, this idea does not work. This is because there is a fundamental difference between the two systems: the existence of solvent at the metal-liquid interface at the electrode. In electrochemistry, an electric dipole layer is formed at the electrode surface when it is biased. Thus, a large electric field gradient appears across the dipole layer, whereas no such steep electric field gradients exist at metal surfaces in vacuum, unless a counter electrode is located at very close to the metal surface. Thus, even if we 
could apply a bias voltage pulse with a fs rising edge to the electrode, it takes a much longer time to fill charges at the metal electrode-liquid interface to establish the dipole layer with an electric field gradient.

Considering this similarity and dissimilarity between metal surfaces in vacuum and interfaces between metal and liquid, it is interesting to see what happens if electrons in a metal electrode are impulsively excited by ultrashort laser pulses. At metal surfaces with adsorbates in UHV, desorption seems to be a major pathway for adsorbates. When adsorbates are placed at a metal-liquid interface, the situation is very different. Although the external modes of adsorbate will be excited as in the case of adsorbate on metal in vacuum, adsorbate has a large potential barrier to depart from the interface, because it is surrounded by solvent molecules. Thus, adsorbate is retained at the interface, while substrate electrons are highly excited. This type of cage effect may open a new reaction pathway. Moreover, ultrafast dynamics induced by short laser pulses at electrode surfaces may be useful to clarify a role of solvent in nonthermal reactions at metal-liquid interfaces; this is a crucial factor to bridge the pressure gap between surface science dealing with surfaces in UHV and electrochemistry dealing with metal-liquid interfaces.

\section{B. Photocatalysis at semiconductor-liquid interfaces}

One of the biggest challenges in surface photochemistry is to understand the mechanism of photocatalysis at the molecular level. Among photocatalytic reactions, it is fascinating to explore the mechanism of water splitting particularly with a powdered photocatalyst of metal oxide under visible light irradiation ${ }^{38}$ because of the following reasons. First, water splitting is not only scientifically interesting, but also important in the applied field. This is one form of artificial photosynthesis: ${ }^{39}$ solar energy is converted to chemical energy by producing molecules that can be used as a storage of energy and reused to extract energy when needed. Second, the photocatalysis with metal oxides is composed of many fundamental processes: electronic excitation of semiconductor photocatalysts, charge splitting, transportation of charges to photocatalyst surfaces, and redox reactions at the surfaces. Thus, the photocatalysis contains a variety of ingredients in physics and chemistry: the photocatalysts of metal oxide are highly integrated systems. Consequently, the photocatalysis raises very interesting scientific questions across many disciplines.

Actually the history of photocatalytic reactions with semiconductors is older than the "surface photochemistry" at metal surfaces in UHV discussed in this paper. Since the epoch making work by Honda and Fujishima in 1972 on the electrochemical photolysis of water with titania, ${ }^{40}$ a large amount of studies has been conducted. ${ }^{41,42}$ The surface science approach is to use a single crystal of photocatalyst under UHV conditions. With this method the surface structure and adsorbates can be well characterized. In fact, many works have been done particularly on rutile $\mathrm{TiO}_{2}$ single crystals. ${ }^{43-45}$ However, this approach has serious problems: the pressure and materials gaps. These are not inherent in photocatalysis; these are well-known problems in surface science for understanding realistic catalytic reactions that are operated under high pressures and with catalysts in the forms of powders, pellets, etc.

In spite of the extensive works on photocatalysis with semiconductors, there are a number of problems to be clarified for a better understanding of the mechanisms of photocatalysis. This is because the integrated systems, such as powdered photocatalysts, are very complex. Among many problems, I will point out only a couple of important problems here regarding charge separation. Let us focus on photocatalytic reactions in liquid such as water splitting with semiconductor powdered catalysts. Photocatalysis of powdered catalysts can be viewed as "back-to-back" or short-circuited photochemical and electrochemical reactions. ${ }^{42}$ Electron-hole pairs are produced in a particle of photocatalyst as a result of optical transition across the bandgap of the photocatalyst. Charges are separated and transported to its surfaces, generating electric fields at the interface with water. Then, redox reactions at the interface proceed under the photovoltage. Now important questions are in the following: what is responsible for charge separation and how large and to what direction do local electric fields point at the photocatalyst-water interfaces?

Charge separation is of paramount importance in photocatalysis with powdered catalysts. This is one of the most crucial factors that determine the efficiency of photocatalyst. Because powdered photocatalysts do not have a $p, n$-junction as in photodiodes, there are no internal electric field gradients that drive charge separation of electron-hole pairs generated. We can understand the charge separation of bulk semiconductors in terms of electric field gradients due to the band bending of valence and conduction bands at surfaces. Electrons or holes are preferentially accumulated, depending on which direction the bands bend. This charge separation mechanism due to band bending may not be applicable to powdered photocatalysts. The width of a space charge layer associated with the band bending depends on the dielectric constant of photocatalyst, voltage, and carrier density at the surface, etc. Assuming the carrier density of $10^{15}-10^{16} \mathrm{~cm}^{-3}$ in rutile $\mathrm{TiO}_{2}$, the space charge layer ranges from 1 to $4 \mu \mathrm{m}$; this is wider than or almost compatible to the typical size of powdered photocatalysts. Thus, it is not clear whether or not we can understand the charge separation in terms of band bending.

It is interesting to note the facet dependence of redox reactions in powdered photocatalysts. $\mathrm{PbO}_{2}$ was oxidatively deposited from $\mathrm{Pb}^{2+}$ mainly on (110) faces of rutile $\mathrm{TiO}_{2}$ particles, while $\mathrm{Pt}$ is reductively deposited from $\mathrm{PtCl}_{6}^{2-}$ on (011) faces. ${ }^{46}$ The similar facet dependent photodeposition of metals was observed on other titanate photocatalysts with a (111) plane-type perovskite structure. ${ }^{47}$ Because the photodeposition of metals depends not only on the voltage created by charges accumulated at the interface but also on the adsorption energy of ions, these results do not necessarily provide the direct evidence of preferential accumulation of electrons or holes at different facets. Thus, it is necessary to measure directly how surface photovoltage depends on the structure of facets of photocatalyst crystallites. Then, we will have more solid knowledge how the geometric structure at the surface and subsurface of crystallites determines the direction and 
magnitude of surface dipoles and how these surface dipoles affect the efficiency of charge splitting and the selectivity in charge accumulation at the interfaces of photocatalysts.

Charge trapping sites, important for redox reactions to occur effectively, are also relevant to charge separation and accumulation of charges at photocatalysts surfaces. Charges are stabilized and localized at some surface sites. Trapped charges should not be stabilized too much, because charges localized in deep traps loose redox power, while the potential barrier for charge transfer at the interface increases. Distributions of trapping sites also depend on the crystal facet structure. Thus, it is crucial to characterize and measure the facet dependence of trapping sites, in particular, shallow traps that are expected to play an important role in charge transfer at the solid-liquid interfaces of photocatalysts.

\section{CONCLUDING REMARKS}

In this paper, I described the mechanisms of electronic excitation that initiates nuclear dynamics of adsorbates. The adsorption system of $\mathrm{Cs}$ on $\mathrm{Cu}(111)$ is a good example to show different types of excitation and how the electronic excitation stimulates adsorbate nuclear dynamics. Experimental and theoretical techniques will be sophisticated in near future; they allow us to understand and predict photoinduced nuclear dynamics at surfaces in higher precision.

In addition to such sophistication in the studies of photochemistry of well-defined adsorption systems, much effort has to be devoted to more complicated systems. Photochemistry and photoinduced nuclear dynamics at liquid-solid interfaces are one example. More serious challenges are in integrated heterogeneous systems. I have pointed out the problems regarding separation and accumulation of charges at surfaces of crystallite photocatalysts. These are, however, the primary steps of photocatalysis. Many processes follow the steps: under the electric field induced by charges accumulated, reactant, and solvent molecules at the interface of photocatalyst will reorient; charge transfer at the interface occurs when the configuration of reactant is properly met; then, surface reactions really proceed via reaction intermediates. Many fundamental processes have to be disentangled and each step has to be explored in detail. The road to the final goal, i.e., a total understanding of photocatalysis, is still rough. However, these systems provide a good opportunity to extend our knowledge of surface photochemistry.

\section{ACKNOWLEDGMENTS}

I would like to thank my collaborators and students, in particular, Kazuya Watanabe, who were involved in the project of surface photochemistry and photoinduced surface dynamics in my group.

${ }^{1}$ Laser Spectroscopy and Photochemistry on Metal Surfaces, edited by H.-L. Dai and W. Ho, Advanced Series in Physical Chemistry Vol. 5 (World Scientific, Singapore, 1995).

${ }^{2}$ Y. A. Gruzdkov, K. Watanabe, K. Sawabe, and Y. Matsumoto, Chem. Phys. Lett. 227, 243 (1994).
${ }^{3}$ K. Watanabe, K. Sawabe, and Y. Matsumoto, Phys. Rev. Lett. 76, 1751 (1996).

${ }^{4}$ Y. Matsumoto, Y. A. Gruzdkov, K. Watanabe, and K. Sawabe, J. Chem. Phys. 105, 4775 (1996).

${ }^{5}$ Y. Matsumoto, Bull. Chem. Soc. Jpn. 80, 842 (2007).

${ }^{6} \mathrm{H}$. Petek and J. Zhao, Chem. Rev. 110, 7082 (2010).

${ }^{7}$ H. Öström, H. Ogasawara, L.-A. Näslund, K. Andersson, L. G. M. Pettersson, and A. Nilsson, J. Chem. Phys. 127, 144702 (2007).

${ }^{8}$ H. P. Bonzel, Physics and Chemistry of Alkali Metal Adsorption, edited by A. M. Bradshaw and G. Ertl (Elsevier, Amsterdam, 1989).

${ }^{9}$ N. Fischer, S. Schuppler, T. Fauster, and W. Steinmann, Surf. Sci. 314, 89 (1994).

${ }^{10}$ J. M. Carlsson and B. Hellsing, Phys. Rev. B 61, 13973 (2000).

${ }^{11}$ F. Schiller, M. Corso, M. Urdanpilleta, T. Ohta, A. Bostwick, J. L. McChesney, E. Rotenberg, and J. E. Ortega, Phys. Rev. B 77, 153410 (2008).

${ }^{12}$ J. P. Gauyacq, A. G. Borisov, and M. Bauer, Prog. Surf. Sci. 82, 244 (2007).

${ }^{13}$ J. Zhao, N. Pontius, A. Winkelmann, V. Sametoglu, A. Kubo, A. G. Borisov, D. Sanchez-Portal, V. M. Silkin, E. V. Chulkov, P. M. Echenique, and H. Petek, Phys. Rev. B 78, 085419 (2008)

${ }^{14}$ E. Wimmer, J. Phys. F: Met. Phys. 13, 2313 (1983).

${ }^{15}$ H. Petek, M. J. Weida, H. Nagano, and S. Ogawa, Science 288, 1402 (2000).

${ }^{16}$ H. Petek, H. Nagano, M. J. Weida, and S. Ogawa, J. Phys. Chem. B 105, 6767 (2001).

${ }^{17}$ Y. Matsumoto and K. Watanabe, Chem. Rev. 106, 4234 (2006).

${ }^{18} \mathrm{~K}$. Watanabe, Y. Matsumoto, T. Yasuike, and K. Nobusada, J. Phys. Chem. A 115, 9528 (2011).

${ }^{19}$ D. A. Arena, F. G. Curti, and R. A. Bartynski, Phys. Rev. B 56, 15404 (1997).

${ }^{20}$ V. Chis, S. Caravati, G. Butti, M. I. Trioni, P. Cabrera-Sanfelix, A. Arnau, and B. Hellsing, Phys. Rev. B 76, 153404 (2007).

${ }^{21}$ J. Gadzuk, Chem. Phys. Lett. 80, 5 (1981).

${ }^{22}$ D. C. Langreth, Phys. Rev. Lett. 54, 126 (1985).

${ }^{23}$ Y. J. Chabal, Surf. Sci. Rep. 8, 211 (1988).

${ }^{24}$ E. J. Heilweil, M. P. Casassa, R. R. Cavanagh, and J. C. Stephenson, Annu. Rev. Phys. Chem. 40, 143 (1989).

${ }^{25}$ R. R. Cavanagh, E. J. Heilweil, and J. C. Stephenson, Surf. Sci. 299/300, 643 (1994).

${ }^{26}$ J. D. Beckerle, "Spectroscopy and dynamics of vibrationally excited adsorbates on metal surfaces," in Laser Spectroscopy and Photochemistry on Metal Surfaces, edited by H.-L. Dai and W. Ho (World Scientific, Singapore, 1995), Chap. 12, pp. 459-497.

${ }^{27}$ H. Ueba, Prog. Surf. Sci. 55, 115 (1997).

${ }^{28}$ M. Brandbyge, P. Hedegård, T. F. Heinz, J. A. Misewich, and D. M. Newns, Phys. Rev. B 52, 6042 (1995).

${ }^{29}$ E. H. G. Backus, A. Eichler, A. W. Kleyn, and M. Bonn, Science 310, 1790 (2005).

${ }^{30}$ H. Watanabe, S. Yamaguchi, S. Sobhan, A. Morita, and T. Tahara, J. Chem. Phys. 132, 144701 (2010).

${ }^{31}$ F. Kao, D. Busch, D. G. da Costa, and W. Ho, Phys. Rev. Lett. 70, 4098 (1993).

${ }^{32}$ W. D. Mieher and W. Ho, J. Chem. Phys. 99, 9279 (1993).

${ }^{33}$ M. Bonn, S. Funk, C. Hess, D. N. Denzler, C. Stempfl, M. Scheffler, M. Wolf, and G. Ertl, Science 285, 1042 (1999).

${ }^{34}$ K. Sawabe, J. Lee, and Y. Matsumoto, J. Chem. Phys. 99, 3143 (1993).

${ }^{35}$ C. Frischkorn and M. Wolf, Chem. Rev. 106, 4207 (2006).

${ }^{36}$ P. Nuernberger, D. Wolpert, H. Weiss, and G. Gerber, Proc. Natl. Acad. Sci. U.S.A. 107, 10366 (2010).

${ }^{37}$ P. Nuernberger, D. Wolpert, H. Weiss, and G. Gerber, Phys. Chem. Chem. Phys. 14, 1185 (2012).

${ }^{38}$ A. Kudo and Y. Miseki, Chem. Soc. Rev. 38, 253 (2009).

${ }^{39}$ A. J. Bard and M. A. Fox, Acc. Chem. Res. 28, 141 (1995).

${ }^{40}$ A. Fujishima and K. Honda, Nature (London) 238, 37 (1972).

${ }^{41}$ A. Fujishima, T. N. Rao, and D. A. Tryk, J. Photochem. Photobiol. C 1, 1 (2000).

${ }^{42}$ A. Fujishima, X. Zhang, and D. A. Tryk, Surf. Sci. Rep. 63, 515 (2008).

${ }^{43}$ A. L. Linsebigler, G. Lu, and J. T. Yates, Jr., Chem. Rev. 95, 735 (1995).

${ }^{44}$ T. Thompson and J. T. Yates, Jr., Chem. Rev. 106, 4428 (2006).

${ }^{45}$ M. A. Henderson, Surf. Sci. Rep. 66, 185 (2011).

${ }^{46}$ T. Ohno, K. Sarukawa, and M. Matsumura, New J. Chem. 26, 1167 (2002).

${ }^{47}$ Y. Miseki, H. Kato, and A. Kudo, Energy Environ. Sci. 2, 306 (2009). 